Volume 132, Number 9, Pages 2753-2759

S 0002-9939(04)07449-0

Article electronically published on April 21, 2004

\title{
MOMENT GENERATING FUNCTION OF THE RECIPROCAL OF AN INTEGRAL OF GEOMETRIC BROWNIAN MOTION
}

\author{
KYOUNGHEE KIM
}

(Communicated by Richard C. Bradley)

\begin{abstract}
In this paper we obtain a simple, explicit integral form for the moment generating function of the reciprocal of the random variable defined by $A_{t}^{(\nu)}:=\int_{0}^{t} \exp \left(2 B_{s}+2 \nu s\right) d s$, where $B_{s}, s>0$, is a one-dimensional Brownian motion starting from 0 . In case $\nu=1$, the moment generating function has a particularly simple form.
\end{abstract}

\section{INTRODUCTION}

Recently, many mathematicians have been interested in the distribution of integrals of geometric Brownian motion, which arise in the field of mathematical finance and are related to the average value option, an Asian option.

The stochastic process

$$
A_{t}^{(\nu)}:=\int_{0}^{t} \exp \left(2 B_{s}+2 \nu s\right) d s
$$

where $B_{s}, s>0$, is a one-dimensional Brownian motion starting from 0 , appears in many areas involving geometric Brownian motions. This process has manifold applications, ranging from the physics of random media to mathematical finance. In mathematical finance, valuing the Asian options asks for as explicit an expression as possible for a certain functional of the integral of geometric Brownian motion over a finite time interval. Previous work on the moment generating function of $A_{t}^{(\nu)}$ by Yor has produced an implicit formula which is difficult to use (see equation (7.e) in [6]). Our formula for the moment generating function of the reciprocal of $A_{t}^{(\nu)}$ is easier to integrate or differentiate. We can obtain positive and negative moments directly from the result.

Girsanov's theorem lets us obtain, for any Borel function $f: \mathbb{R}_{+} \rightarrow \mathbb{R}_{+}$,

$$
\mathbb{E}\left[f\left(A_{t}^{(\nu)}\right)\right]=\mathbb{E}\left[f\left(A_{t}\right) \exp \left(\nu B_{t}-\frac{1}{2} \nu^{2} t\right)\right]
$$

where $A_{t}$ is the case $\nu=0$.

Received by the editors December 13, 2002 and, in revised form, July 18, 2003.

2000 Mathematics Subject Classification. Primary 60J65; Secondary 60G35.

Key words and phrases. Geometric Brownian motion, Asian options, moment generating functions.

(C)2004 American Mathematical Society 
In 2] Bougerol obtained the joint distribution of $\left(\int_{0}^{t} e^{B_{s}} d W_{s}, \exp \left(B_{t}\right)\right)$ for $t>0$ (where $B_{t}$ is a real-valued Brownian motion starting from 0 and $W_{t}$ is an independent $\mathbb{R}^{d}$-valued Brownian motion starting from the origin), in terms of the semigroup of the hyperbolic Brownian motion on Poincaré's half-plane. An interesting identity is also obtained in his paper:

$$
\text { for any fixed } t>0, \quad \sinh \left(B_{t}\right)=\gamma_{A_{t}} \text { in law, }
$$

where $\left(\gamma_{t}, t \geq 0\right)$ is a one-dimensional Brownian motion starting at 0 , and independent of $B_{t}, t \geq 0$. Using this surprising identity, the integer moments of $A_{t}$ are computed by Yor 6 .

The integral form solution of the conditional expectation

$$
\mathbb{E}\left[\frac{1}{1+2 \int_{0}^{T} e^{2\left(B_{s}+s\right)} d s} \mid \mathcal{F}_{t}\right], \quad T \geq t,
$$

is computed by Goodman and Kim [4]. We invert the Laplace transform obtained in Section 3 to derive the moment generating function of the reciprocal of $A_{t}$, by comparing a Laplace transform with the result in [4].

Main Theorem. For any $\beta \geq 0$ and any $t>0$,

$$
\begin{gathered}
\mathbb{E}\left[\exp \left(-\frac{1}{2 A_{t}^{(1)}} \sinh ^{2} \beta\right)\right]=f(t) \int_{\beta}^{\infty} 2 r e^{-\frac{r^{2}}{2 t}} \sqrt{\sinh ^{2} r-\sinh ^{2} \beta} d r, \\
\text { where } f(t)=\frac{e^{-\frac{1}{2} t}}{t \sqrt{2 \pi t}} .
\end{gathered}
$$

The paper is organized as follows. In the next section we derive the identity which leads us to the Laplace transform for the reciprocal of $A_{t}$. The expectation of $\frac{1}{1+2 s A_{t}^{(1)}}$ is in the following section. The moment generating function is then an immediate consequence. The positive moments of $A_{t}^{(1)}$ are provided in the last section.

\section{LAPLACE TRANSFORM OF THE MOMENT GENERATING FUNCTION OF MINUS THE RECIPROCAL OF $A_{t}^{(1)}$}

Let

$$
M(u):=\mathbb{E}\left[\exp \left(-\frac{1}{2 A_{t}^{(1)}} u\right)\right], \quad u \geq 0 .
$$

The Laplace transform of $M(u)$ is given by

$$
\mathcal{M}(s)=\int_{0}^{\infty} M(u) e^{-s u} d u, \quad s \geq 0,
$$

and therefore by reversing the order of integration we have

$$
\begin{aligned}
\mathcal{M}(s) & =\mathbb{E}\left[\int_{0}^{\infty} \exp \left(-\frac{1}{2 A_{t}^{(1)}} u\right) e^{-s u} d u\right] \\
& =\mathbb{E}\left[\frac{1}{s+\frac{1}{2 A_{t}^{(1)}}}\right] \\
& =\frac{1}{s}\left(1-\mathbb{E}\left[\frac{1}{1+2 s A_{t}^{(1)}}\right]\right) .
\end{aligned}
$$


We now define

$$
\begin{aligned}
N(u) & :=-\frac{d M(u)}{d u} \\
& =\mathbb{E}\left[\frac{1}{2 A_{t}^{(1)}} \exp \left(-\frac{1}{2 A_{t}^{(1)}} u\right)\right] .
\end{aligned}
$$

Using an identity for the Laplace transform of a derivative and (2.3), we obtain

$$
\begin{aligned}
\mathcal{N}(s) & =\int_{0}^{\infty} N(u) e^{-s u} d u, \quad s \geq 0, \\
& =\mathbb{E}\left[\frac{1}{1+2 s A_{t}^{(1)}}\right] .
\end{aligned}
$$

\section{Expectation of a Certain function of $A_{t}^{(1)}$}

As we remarked in the Introduction, we have the joint density of $\left(\int_{0}^{t} e^{B_{s}} d W_{s}, \exp \left(B_{t}\right)\right)$ (Bougerol [2]). In the case that $\left(W_{t}, t>0\right)$ is $\mathbb{R}^{2}$-valued Brownian motion, this joint density is quite simple. For $z \in \mathbb{R}^{2}$ and $x \in \mathbb{R}$, the joint density is given by

$$
\begin{aligned}
& p_{t}\left(z, e^{x}\right)=e^{-x} \frac{r}{\sinh r} \frac{1}{(2 \pi t)^{\frac{3}{2}}} e^{-\frac{r^{2}}{2 t}}, \\
& \text { where } r=\operatorname{Arg} \cosh \left[\cosh x+\frac{e^{-x}\|z\|^{2}}{2}\right] .
\end{aligned}
$$

We now remark that for a deterministic function $g(t)$, it is well known that $\int_{0}^{t} g(s) d W_{s}$ has the Gaussian distribution with mean 0 and variance $\int_{0}^{t} g^{2}(s) d s$. In particular,

$$
\int_{0}^{t} g(s) d W_{s} \stackrel{d}{=}\left(\int_{0}^{t} g^{2}(s) d s\right)^{\frac{1}{2}} \cdot W_{1}
$$

and

$$
\mathbb{E}\left[\left(\int_{0}^{t} g(s) d W_{s}\right)^{2}\right]=\mathbb{E}\left[\int_{0}^{t} g^{2}(s) d s \cdot W_{1}^{2}\right]
$$

As a consequence, we get

$$
\mathbb{E}\left[A_{t}\left(W_{1}\right)^{2} \mid A_{t}, B_{t}\right]=\mathbb{E}\left[\left(\int_{0}^{t} e^{B_{s}} d W_{s}\right)^{2} \mid A_{t}, B_{t}\right],
$$

where $\left(W_{s}, s \geq 0\right)$ is a one-dimensional Brownian motion starting at 0 , and independent of $B$.

Using (3.3) and (1.2), we have

$$
\begin{aligned}
\mathbb{E}\left[\frac{1}{1+2 s A_{t}^{(1)}}\right] & =e^{-\frac{1}{2} t} \mathbb{E}\left[e^{B_{t}} \frac{1}{1+2 s A_{t}}\right] \\
& =e^{-\frac{1}{2} t} \mathbb{E}\left[e^{B_{t}} \mathbb{E}\left[\frac{1}{1+2 s A_{t}} \mid A_{t}, B_{t}\right]\right] \\
& =e^{-\frac{1}{2} t} \mathbb{E}\left[e^{B_{t}} \mathbb{E}\left[e^{-s A_{t}\left(\left(W_{1}\right)^{2}+\left(W_{1}^{\prime}\right)^{2}\right)} \mid A_{t}, B_{t}\right]\right] \\
& =e^{-\frac{1}{2} t} \mathbb{E}\left[e^{B_{t}} \mathbb{E}\left[e^{-s\left(\left(\int_{0}^{t} e^{B_{s}} d W_{s}\right)^{2}+\left(\int_{0}^{t} e^{B_{s}} d W_{s}^{\prime}\right)^{2}\right)} \mid A_{t}, B_{t}\right]\right] \\
& =e^{-\frac{1}{2} t} \mathbb{E}\left[e^{B_{t}} e^{-s\left(\left(\int_{0}^{t} e^{B_{s}} d W_{s}\right)^{2}+\left(\int_{0}^{t} e^{B_{s}} d W_{s}^{\prime}\right)^{2}\right)}\right]
\end{aligned}
$$


where $\left(\left(W_{t}, W_{t}^{\prime}\right), t \geq 0\right)$ is a two-dimensional Brownian motion starting from the origin, and independent of $B$.

Using the joint density (3.1) and polar coordinates, we have

$$
\begin{gathered}
\mathbb{E}\left[e^{B_{t}} e^{-s\left(\left(\int_{0}^{t} e^{B_{s}} d W_{s}\right)^{2}+\left(\int_{0}^{t} e^{B_{s}} d W_{s}^{\prime}\right)^{2}\right)}\right] \\
=\frac{1}{t \sqrt{2 \pi t}} \int_{-\infty}^{\infty} \int_{0}^{\infty} e^{-s \rho^{2}} \frac{r}{\sinh r} e^{-\frac{r^{2}}{2 t}} \rho d \rho d x \\
\text { where } \quad r=\operatorname{Arg} \cosh \left[\cosh x+\frac{e^{-x} \rho^{2}}{2}\right] .
\end{gathered}
$$

After a change of variables, the equation (3.5) becomes

$$
\begin{aligned}
\frac{1}{t \sqrt{2 \pi t}} & \int_{-\infty}^{\infty} \int_{0}^{\infty} e^{-s \rho^{2}} \frac{r}{\sinh r} e^{-\frac{r^{2}}{2 t}} \rho d \rho d x \\
& =\frac{1}{t \sqrt{2 \pi t}} \int_{0}^{\infty} \int_{-r}^{r} e^{-2 s e^{x}(\cosh r-\cosh x)} r e^{-\frac{r^{2}}{2 t}} e^{x} d x d r .
\end{aligned}
$$

Notice that

$$
2 e^{x}(\cosh r-\cosh x)=-\left(e^{x}-\cosh r\right)^{2}+\sinh ^{2} r .
$$

Using $u=e^{x}-\cosh r$, we can rewrite (3.6) more simply:

$$
\begin{aligned}
\frac{1}{t \sqrt{2 \pi t}} \int_{0}^{\infty} & \int_{-r}^{r} e^{-2 s e^{x}(\cosh r-\cosh x)} r e^{-\frac{r^{2}}{2 t}} e^{x} d x d r \\
& =\frac{1}{t \sqrt{2 \pi t}} \int_{0}^{\infty} \int_{-\sinh r}^{\sinh r} r e^{-\frac{r^{2}}{2 t}} e^{-s\left(\sinh ^{2} r-u^{2}\right)} d u d r \\
& =\frac{2}{t \sqrt{2 \pi t}} \int_{0}^{\infty} r e^{-\frac{r^{2}}{2 t}} e^{-s \sinh ^{2} r}\left(\int_{0}^{\sinh r} e^{s u^{2}} d u\right) d r .
\end{aligned}
$$

From (3.4) and (3.7), we can deduce the following result.

Theorem 1 (Goodman and Kim [4]). For any $t>0$ and for any $s \in \mathbb{R}$, we have the formula

$$
\mathbb{E}\left[\frac{1}{1+2 s A_{t}^{(1)}}\right]=\frac{2}{t \sqrt{2 \pi t}} e^{-\frac{t}{2}} \int_{0}^{\infty} r e^{-\frac{r^{2}}{2 t}} e^{-s \sinh ^{2} r}\left(\int_{0}^{\sinh r} e^{s v^{2}} d v\right) d r .
$$

Making a change of variables in (3.8), we obtain

$$
\mathbb{E}\left[\frac{1}{1+2 s A_{t}^{(1)}}\right]=\int_{0}^{\infty} \int_{\operatorname{Arg} \sinh \sqrt{u}}^{\infty} \frac{1}{t \sqrt{2 \pi t}} r e^{-\frac{r^{2}}{2 t}} e^{-\frac{t}{2}} \frac{1}{\sqrt{\sinh ^{2} r-u}} d r e^{-s u} d u
$$

Now we define

$$
g(u)=\int_{\operatorname{Arg} \sinh \sqrt{u}}^{\infty} \frac{1}{t \sqrt{2 \pi t}} r e^{-\frac{r^{2}}{2 t}} e^{-\frac{t}{2}} \frac{1}{\sqrt{\sinh ^{2} r-u}} d r .
$$

Therefore, we arrive at another Laplace transform:

$$
\mathbb{E}\left[\frac{1}{1+2 s A_{t}^{(1)}}\right]=\int_{0}^{\infty} g(u) e^{-s u} d u .
$$




\section{Moment Generating FUnCtion}

We now consider the moment generating function of the reciprocal of $A_{t}^{(1)}$. In the previous sections, we obtained two different inverse Laplace transforms of $\mathbb{E}\left[\frac{1}{1+2 s A_{t}^{(1)}}\right]$, namely (2.5) and (3.10). The injectivity of the Laplace transform gives us the following result:

$$
\mathbb{E}\left[\frac{1}{2 A_{t}^{(1)}} \exp \left(-\frac{1}{2 A_{t}^{(1)}} u\right)\right]=\int_{\operatorname{Arg} \sinh \sqrt{u}}^{\infty} \frac{1}{t \sqrt{2 \pi t}} r e^{-\frac{r^{2}}{2 t}} e^{-\frac{t}{2}} \frac{1}{\sqrt{\sinh ^{2} r-u}} d r .
$$

Let

$$
\sqrt{u}=\sinh \theta, \quad u \geq 0 .
$$

Then our equation becomes

$$
\mathbb{E}\left[\frac{1}{2 A_{t}^{(1)}} \exp \left(-\frac{1}{2 A_{t}^{(1)}} \sinh ^{2} \theta\right)\right]=\int_{\theta}^{\infty} \frac{1}{t \sqrt{2 \pi t}} r e^{-\frac{r^{2}}{2 t}} e^{-\frac{t}{2}} \frac{1}{\sqrt{\sinh ^{2} r-\sinh ^{2} \theta}} d r
$$

To get the desired moment generating function, we integrate the equation (4.2) from $\beta$ to $\infty$. Introducing the integration factor $2 \sinh \theta \cosh \theta$, we can obtain

$$
\int_{\beta}^{\infty} \mathbb{E}\left[\frac{1}{2 A_{t}^{(1)}} \exp \left(-\frac{1}{2 A_{t}^{(1)}} \sinh ^{2} \theta\right)\right] d \sinh ^{2} \theta=\mathbb{E}\left[\exp \left(-\frac{1}{2 A_{t}^{(1)}} \sinh ^{2} \beta\right)\right] .
$$

Also, we have

$$
\begin{aligned}
\int_{\beta}^{\infty} \int_{\theta}^{\infty} & \frac{1}{t \sqrt{2 \pi t}} r e^{-\frac{r^{2}}{2 t}} e^{-\frac{t}{2}} \frac{1}{\sqrt{\sinh ^{2} r-\sinh ^{2} \theta}} d r d \sinh ^{2} \theta \\
& =\frac{e^{-\frac{1}{2} t}}{t \sqrt{2 \pi t}} \int_{\beta}^{\infty}\left[\int_{\beta}^{r} \frac{2 \sinh \theta \cosh \theta}{\sqrt{\sinh ^{2} r-\sinh ^{2} \theta}} d \theta\right] r e^{-\frac{r^{2}}{2 t}} d r
\end{aligned}
$$

Making some changes of variables, we obtain

$$
\int_{\beta}^{r} \frac{2 \sinh \theta \cosh \theta}{\sqrt{\sinh ^{2} r-\sinh ^{2} \theta}} d \theta=2 \sqrt{\sinh ^{2} r-\sinh ^{2} \beta} .
$$

Using (4.5), we obtain

$$
\begin{aligned}
\frac{e^{-\frac{1}{2} t}}{t \sqrt{2 \pi t}} \int_{\beta}^{\infty} & {\left[\int_{\beta}^{r} \frac{2 \sinh \theta \cosh \theta}{\sqrt{\sinh ^{2} r-\sinh ^{2} \theta}} d \theta\right] r e^{-\frac{r^{2}}{2 t}} d r } \\
& =\frac{e^{-\frac{1}{2} t}}{t \sqrt{2 \pi t}} \int_{\beta}^{\infty} 2 r e^{-\frac{r^{2}}{2 t}} \sqrt{\sinh ^{2} r-\sinh ^{2} \beta} d r .
\end{aligned}
$$

Consequently, we deduce from (4.3) and (4.6) that

$$
\mathbb{E}\left[\exp \left(-\frac{1}{2 A_{t}^{(1)}} \sinh ^{2} \beta\right)\right]=\frac{e^{-\frac{1}{2} t}}{t \sqrt{2 \pi t}} \int_{\beta}^{\infty} 2 r e^{-\frac{r^{2}}{2 t}} \sqrt{\sinh ^{2} r-\sinh ^{2} \beta} d r .
$$

Similar computations allow us to obtain the moment-generating functions of the reciprocal of $A_{t}^{(\nu)}$ for any $\nu \in \mathbb{R}^{*}$, where $\mathbb{R}^{*}$ is the set of non-zero real numbers. 
Theorem 2. For any $\nu \in \mathbb{R}^{*}$, for any $\beta \geq 0$, and for any $t>0$, we have

$$
\begin{gathered}
\mathbb{E}\left[\exp \left(-\frac{1}{2 A_{t}^{(\nu)}} \sinh ^{2} \beta\right)\right]=f^{(\nu)}(t) \int_{\beta}^{\infty} \xi_{\beta}^{(\nu)}(r) r e^{-\frac{r^{2}}{2 t}} d r, \\
\text { where } f^{(\nu)}(t)=\frac{1}{t \sqrt{2 \pi t}} e^{-\frac{\nu^{2} t}{2}}, \\
\xi_{\beta}^{(\nu)}(r)=\frac{1}{\nu}\left[\left(\cosh r+\sqrt{\sinh ^{2} r-\sinh ^{2} \beta}\right)^{\nu}\right. \\
\left.-\left(\cosh r-\sqrt{\sinh ^{2} r-\sinh ^{2} \beta}\right)^{\nu}\right] .
\end{gathered}
$$

5. The Moments of $A_{t}^{(1)}$

The iterated integration of the moment generating function for the reciprocal of $A_{t}^{(1)}$ leads us the positive moments of $A_{t}^{(1)}$.

Multiplying (4.7) by $2 \sinh \beta \cosh \beta$, we obtain

$$
\begin{aligned}
\mathbb{E}[\exp ( & \left.\left.-\frac{1}{2 A_{t}^{(1)}} \sinh ^{2} \beta\right) 2 \sinh \beta \cosh \beta\right] \\
\quad= & \frac{e^{-\frac{1}{2} t}}{t \sqrt{2 \pi t}} \int_{\beta}^{\infty} 2 r e^{-\frac{r^{2}}{2 t}} 2 \sinh \beta \cosh \beta \sqrt{\sinh ^{2} r-\sinh ^{2} \beta} d r .
\end{aligned}
$$

Integrating (5.1) from $\gamma$ to $\infty$, we have the following result:

$$
\begin{aligned}
2 \mathbb{E}[ & \left.A_{t}^{(1)} \exp \left(-\frac{1}{2 A_{t}^{(1)}} \sinh ^{2} \gamma\right)\right] \\
& =\frac{2 e^{-\frac{1}{2} t}}{t \sqrt{2 \pi t}} \int_{\gamma}^{\infty} \int_{\beta}^{\infty} \sqrt{\sinh ^{2} r-\sinh ^{2} \beta} r e^{-\frac{r^{2}}{2 t}} d r d \sinh ^{2} \beta \\
& =\frac{2 e^{-\frac{1}{2} t}}{t \sqrt{2 \pi t}} \int_{\gamma}^{\infty} \int_{\gamma}^{r} \sqrt{\sinh ^{2} r-\sinh ^{2} \beta} d \sinh ^{2} \beta r e^{-\frac{r^{2}}{2 t}} d r \\
& =\frac{2 e^{-\frac{1}{2} t}}{t \sqrt{2 \pi t}} \int_{\gamma}^{\infty} \frac{2}{3}\left(\sinh ^{2} r-\sinh ^{2} \gamma\right)^{\frac{3}{2}} r e^{-\frac{r^{2}}{2 t}} d r .
\end{aligned}
$$

Integration by parts allows us to have, using (5.2) for $\gamma=0$,

$$
\begin{aligned}
\mathbb{E}\left[A_{t}^{(1)}\right] & =\frac{2 e^{-\frac{1}{2} t}}{\sqrt{2 \pi t}} \int_{0}^{\infty} \cosh r \sinh ^{2} r e^{-\frac{r^{2}}{2 t}} d r \\
& =e^{-\frac{1}{2} t} \mathbb{E}\left[\cosh B_{t}\left(\sinh B_{t}\right)^{2}\right],
\end{aligned}
$$

where $B_{t}$ is a standard Brownian motion starting from 0 .

Repeated integrals give us higher order $A_{t}^{(1)}$ moments. For any $n \in \mathbb{N}$,

$$
\begin{aligned}
\mathbb{E}\left[\left(A_{t}^{(1)}\right)^{n}\right] & =\frac{2 n !}{(2 n) !} e^{-\frac{1}{2} t} \mathbb{E}\left[\cosh B_{t}\left(\sinh B_{t}\right)^{2 n}\right] \\
& =e^{-\frac{1}{2} t} \frac{\mathbb{E}\left[\cosh B_{t}\left(\sinh B_{t}\right)^{2 n}\right]}{\mathbb{E}\left[\left(B_{1}\right)^{2 n}\right]} .
\end{aligned}
$$

Remark. Yor [6] showed that for any $t \geq 0$

$$
\mathbb{E}\left[A_{t}^{n}\right]=\frac{\mathbb{E}\left[\left(\sinh B_{t}\right)^{2 n}\right]}{\mathbb{E}\left[\left(B_{1}\right)^{2 n}\right]}
$$


It seems interesting to compare the two formulas (5.4) and (5.5). Only slight changes are made because of the drift term in $A_{t}^{(1)}$. Girsanov's theorem let us expect the term $e^{-\frac{1}{2} t}$ in (5.4). A different computation for $\mathbb{E}\left[\left(A_{t}^{(1)}\right)^{n}\right]$ can also be found (see section 4 in [6]).

Using Girsanov's theorem allows us to obtain from (5.4), for any $\nu \in \mathbb{R}$ and for any $n \in \mathbb{N}$,

$$
\mathbb{E}\left[\left(A_{t}^{(\nu)}\right)^{n} e^{(1-\nu) B_{t}}\right]=e^{\frac{\nu^{2}}{2} t-\nu t} \frac{\mathbb{E}\left[\cosh B_{t}\left(\sinh B_{t}\right)^{2 n}\right]}{\mathbb{E}\left[\left(B_{1}\right)^{2 n}\right]} .
$$

Using binomial expansion, we deduce the following lemma.

Lemma 1. For any $\nu \in \mathbb{R}$ and for any $n \in \mathbb{N}$,

$$
\begin{aligned}
\mathbb{E} & {\left[\left(A_{t}^{(\nu)}\right)^{n} e^{(1-\nu) B_{t}}\right] } \\
& =e^{\frac{\nu^{2}}{2} t-\nu t} \frac{2 n !}{2^{2 n+1}(2 n) !} \sum_{j=0}^{2 n}\left(\begin{array}{c}
2 n \\
j
\end{array}\right)(-1)^{j}\left(e^{(2 n-2 j-1)^{2} t / 2}+e^{(2 n+2 j+1)^{2} t / 2}\right) .
\end{aligned}
$$

\section{ACKNOWLEDGEMENTS}

The author gives special thanks to Victor W. Goodman for his many valuable suggestions. The author would also like to thank the referee for many helpful comments.

\section{REFERENCES}

[1] R. Bhattacharya, E. Thomann, E. Waymire (2001): A note on the distribution of integrals of geometric Brownian motion, Statist. Probab. Lett. 55, 187-192. MR 2002j:60147

[2] P. Bougerol (1983): Exemples de théorèmes locaux sur les groups résolubles, Ann. Inst. H. Poincaré Sect. B (N.S.) 19, 369-391. MR 84g:60013

[3] H. Geman, M. Yor (1993): Bessel processes, Asian options and perpetuities, Math. Finance 3, 349-375.

[4] V.W. Goodman, K. Kim (2002) : Interest Rate Derivatives within A non-explosive Log Normal Bond Model, working paper

[5] L.C.G. Rogers and Z. Shi (1995): The value of an Asian option, J. Appl. Probab. 32, 10771088. MR 96j:90017

[6] M. Yor (1992): On some exponential functionals of Brownian motion, Adv. in Appl. Probab.24, 509-531. MR 94b:60095

Department of Mathematics, Syracuse University, Syracuse, New York 13244

E-mail address: kkim26@syr.edu 